\title{
Pareceristas 2018-2019
}

Sociologias agradece a colaboração dos consultores ad hoc relacionados a seguir, que gentilmente realizaram pareceres para artigos submetidos entre o último quadrimestre de 2018 e o segundo quadrimestre de 2019.

\begin{tabular}{|c|c|c|c|}
\hline Adriana Garcia Andrade & Universidad Autónoma Metropolitana Azcapotzalco & & México \\
\hline Adriana Paredes-Peñafiel & Universidade Federal do Rio Grande & RS & Brasil \\
\hline Adriano Premebida & Universidade Federal do Rio Grande do Sul & RS & Brasil \\
\hline Alejandra Judith Josiowicz & Fundação Getúlio Vargas & SP & Brasil \\
\hline Amarolinda Klein & Universidade do Vale do Rio dos Sinos & RS & Brasil \\
\hline Ana Cláudia Hebling Meira & Universidade Federal do Espírito Santo & ES & Brasil \\
\hline Ana Paula Comin de Carvalho & Universidade Federal do Recôncavo da Bahia & $\mathrm{BA}$ & Brasil \\
\hline Ana Gonçalves Magalhães & Universidade de São Paulo & SP & Brasil \\
\hline Ana Paula Cavalcanti Simioni & Universidade de São Paulo & SP & Brasil \\
\hline Andrea Fachel Leal & Universidade Federal do Rio Grande do Sul & RS & Brasil \\
\hline Andréa Borges Leão & Universidade Federal do Ceará & CE & Brasil \\
\hline Angela Paiva & Pontifícia Universidade Católica do Rio de Janeiro & RJ & Brasil \\
\hline Antônio Alvaro Zuin & Universidade Federal de São Carlos & SP & Brasil \\
\hline Antônio Brasil Junior & Universidade Federal do Rio de Janeiro & RJ & Brasil \\
\hline Antonio Carlos Silva & Universidade Católica do Salvador & BA & Brasil \\
\hline Antonio David Cattani & Universidade Federal do Rio Grande do Sul & RS & Brasil \\
\hline Antonio Marcos Sanseverino & Universidade Federal do Rio Grande do Sul & RS & Brasil \\
\hline Beatriz Filgueiras & Universidade do Estado do Rio de Janeiro & RJ & Brasil \\
\hline Bianca Ruskowski & Instituto Federal Sulriograndense & RS & Brasil \\
\hline Bruna de Carvalho Lima & Universidade de São Paulo & SP & Brasil \\
\hline Caio Texeira Vasconcellos & Universidade de São Paulo & SP & Brasil \\
\hline Caleb Faria Alves & Universidade Federal do Rio Grande do Sul & RS & Brasil \\
\hline Camila Prates & Universidade Federal do Rio Grande do Sul & RS & Brasil \\
\hline Camila Rocha de Oliveira & Centro Brasileiro de Análise e Planejamento & SP & Brasil \\
\hline Carlos Arturi & Universidade Federal do Rio Grande do Sul & RS & Brasil \\
\hline Carlos Gadea & Universidade do Vale do Rio dos Sinos & RS & Brasil \\
\hline Carlos Steil & Universidade Federal do Rio Grande do Sul & RS & Brasil \\
\hline Carlos Ugo Santander & Universidade Federal de Goiás & $\mathrm{GO}$ & Brasil \\
\hline
\end{tabular}




\begin{tabular}{|c|c|c|c|}
\hline Carolina Bagattolli & Universidade Federal do Paraná & $\mathrm{PR}$ & Brasil \\
\hline Catalina Arteaga & Universidad de Chile & & Chile \\
\hline Catia Grisa & Universidade Federal do Rio Grande do Sul & RS & Brasil \\
\hline Cesar Ceriani Cernadas & Consejo Nacional de Investigaciones Científicas y Técnicas & & Argentina \\
\hline Christiano Tambascia & Universidade Estadual de Campinas & SP & Brasil \\
\hline Cibele Barbosa & Fundação Joaquim Nabuco & PE & Brasil \\
\hline Cibele Saliba Risek & Universidade de São Paulo & $\mathrm{SP}$ & Brasil \\
\hline Claudia Natenzon & Universidad de Buenos Aires & & Argentina \\
\hline Claudia Tirelli & Universidade de Santa Cruz do Sul & RS & Brasil \\
\hline Dani Rudnick & Universidade La Salle - Canoas & RS & Brasil \\
\hline Daniel Guerrini & Universidade Tecnológica Federal do Paraná & PR & Brasil \\
\hline Daniel Mocelin & Universidade Federal do Rio Grande do Sul & RS & Brasil \\
\hline Daniela Alves & Universidade Federal de Viçosa & MG & Brasil \\
\hline Davide Scarso & Universidade Nova de Lisboa & & Portugal \\
\hline Dmitri Fernandes & Universidade Federal de Juiz de Fora & MG & Brasil \\
\hline Edmar Yuta & Faculdades de Campinas & SP & Brasil \\
\hline Edson Silva de Farias & Universidade Estadual do Sudoeste da Bahia & $\mathrm{BA}$ & Brasil \\
\hline Elenice Aparecida Coutinho & Universidade Federal do Rio Grande do Sul & RS & Brasil \\
\hline Emil Sobottka & Pontifícia Universidade Católica do Rio Grande do Sul & RS & Brasil \\
\hline Eveline Rojas & Universidade Federal de Pernambuco & PE & Brasil \\
\hline Fábio Mascaro Querido & Universidade Estadual de Campinas & Sp & Brasil \\
\hline Felipe Gonçalves Brasil & Universidade Estadual Paulista & SP & Brasil \\
\hline Felipe da Luz Colomé & Universidade Federal do Amazonas & AM & Brasil \\
\hline Felipe Vargas & Universidade Federal da Bahia & $\mathrm{BA}$ & Brasil \\
\hline Fernanda Bittencourt Ribeiro & Pontifícia Universidade Católica do Rio Grande do Sul & RS & Brasil \\
\hline Fernando Kulaitis & Universidade Tecnológica Federal do Paraná & $P R$ & Brasil \\
\hline Frederik de Roeck & Ghent University & & Holanda \\
\hline Gabriel Peters & Universidade Federal da Bahia & $\mathrm{BA}$ & Brasil \\
\hline Gabriela Cruz & Universidad de La Republica & & Uruguai \\
\hline Gabriela Lotta & Universidade Federal do $\mathrm{ABC}$ & SP & Brasil \\
\hline Gilson Ciarallo & Universidade de Brasília & DF & Brasil \\
\hline Gloria Baigorrotegui & Universidad de Santiago de Chile & & Chile \\
\hline Guilherme Radomsky & Universidade Federal do Rio Grande do Sul & RS & Brasil \\
\hline Henrique José Amorim & Universidade Federal de São Paulo & SP & Brasil \\
\hline Henrique Zoqui Martins Parra & Universidade Federal de São Paulo & SP & Brasil \\
\hline
\end{tabular}

Sociologias, Porto Alegre, ano 21, n. 52, set-set 2019, p. 314-318. 


\begin{tabular}{|c|c|c|c|}
\hline Ivaldo Gehlen & Universidade Federal do Rio Grande do Sul & RS & Brasil \\
\hline Jane Calvert & University of Edinburgh & & Reino Unido \\
\hline Jean Carlos Hochsprung Miguel & Universidade Federal de São Paulo & SP & Brasil \\
\hline Jean Segata & Universidade Federal do Rio Grande do Norte & $\mathrm{RN}$ & Brasil \\
\hline Jenny Muñoz & Universidade de Passo Fundo & RS & Brasil \\
\hline Jesus Ranieri & Universidade Estadual de Campinas & SP & Brasil \\
\hline José Carlos dos Anjos & Universidade Federal do Rio Grande do Sul & RS & Brasil \\
\hline José Marcos Froehlich & Universidade Federal de Santa Maria & RS & Brasil \\
\hline José Jaime Macedo & Universidade Federal do Vale do São Francisco & $\mathrm{PI}$ & Brasil \\
\hline José Rodrigo Rodriguez & Universidade do Vale do Rio dos Sinos & RS & Brasil \\
\hline José Rogério Lopes & Universidade do Vale do Rio dos Sinos & RS & Brasil \\
\hline Julia Polessa Maçaira & Universidade Federal do Rio de Janeiro & RJ & Brasil \\
\hline Juliana Vicentini & Universidade de São Paulo & SP & Brasil \\
\hline Karl Monsma & Universidade Federal do Rio Grande do Sul & RS & Brasil \\
\hline Léo Peixoto Rodrigues & Universidade Federal de Pelotas & RS & Brasil \\
\hline Leonardo Rafael Santos Leitão & Universidade Federal da Fronteira Sul & SC & Brasil \\
\hline Leticia Schabbach & Universidade Federal do Rio Grande do Sul & RS & Brasil \\
\hline Liliana Acero & Universidad del Pacífico & & Chile \\
\hline Lindomar de Sousa Silva & Empresa Brasileira de Pesquisa Agropecuária & $\mathrm{AM}$ & Brasil \\
\hline Lorena Cândido Fleury & Universidade Federal do Rio Grande do Sul & RS & Brasil \\
\hline Lorena Holzmann & Universidade Federal do Rio Grande do Sul & RS & Brasil \\
\hline Lucas Becerra & Universidad Nacional de Quilmes & & Argentina \\
\hline Luciana Duccini & Universidade Federal da Bahia & $\mathrm{BA}$ & Brasil \\
\hline Luciana Leite Lima & Universidade Federal do Rio Grande do Sul & RS & Brasil \\
\hline Luciana Garcia Mello & Universidade Federal do Rio Grande do Sul & RS & Brasil \\
\hline Luciana Ferreira Tatagiba & Universidade Estadual de Campinas & SP & Brasil \\
\hline Luis Fernando Corrêa da Silva & Universidade Federal do Rio Grande do Sul & RS & Brasil \\
\hline Luiz Antonio Bogo Chies & Universidade Católica de Pelotas & RS & Brasil \\
\hline Luiz Carlos Jackson & Universidade de São Paulo & SP & Brasil \\
\hline Luiz Gustavo Freitas Rossi & Universidade Estadual de Campinas & SP & Brasil \\
\hline Luiz Franscisco A. de Miranda & Universidade Federal de São João Del-Rei & MG & Brasil \\
\hline Luiz Antonio Norder & Universidade Federal de São Carlos & SP & Brasil \\
\hline Marcelo Moura Mello & Universidade Federal da Bahia & $\mathrm{BA}$ & Brasil \\
\hline Marcelo Garson Braule Pinto & Universidade Federal de Juiz de Fora & MG & Brasil \\
\hline Marcia de Paula Leite & Universidade Estadual de Campinas & SP & Brasil \\
\hline
\end{tabular}

Sociologias, Porto Alegre, ano 21, n. 52, set-dez 2019, p. 314-318. 


\begin{tabular}{|c|c|c|c|}
\hline Marco Antonio de Almeida & Universidade de São Paulo & SP & Brasil \\
\hline Marcos Alfonso Spiess & Universidade Federal de Santa Catarina & SC & Brasil \\
\hline Maria Aparecida Bridi & Universidade Federal do Paraná & PR & Brasil \\
\hline Maria Carolina Tomás & Pontifícia Universidade Católica de Minas Gerais & MG & Brasil \\
\hline Maria Cristina Barreto & Universidade do Estado do Rio Grande do Norte & $\mathrm{RN}$ & Brasil \\
\hline Maria Nilza da Silva & Universidade Estadual de Londrina & PR & Brasil \\
\hline Mariana Gomes & Universidade Federal de Santa Maria & RS & Brasil \\
\hline Marilia Luz David & Universidade Federal do Rio Grande do Sul & RS & Brasil \\
\hline Marília Patta Ramos & Universidade Federal do Rio Grande do Sul & RS & Brasil \\
\hline Marisol Marini & Universidade de São Paulo & SP & Brasil \\
\hline Marko Monteiro & Universidade Estadual de Campinas & SP & Brasil \\
\hline Marta Kanashiro & Universidade Estadual de Campinas & SP & Brasil \\
\hline Matheus Mazzilli Pereira & Universidade Federal do Rio Grande do Sul & RS & Brasil \\
\hline Mathilde Navazo & Università degli Studi di Milano & & Italia \\
\hline Mauricio Berger & Universidad de Buenos Aires & & Argentina \\
\hline Maycon Schubert & Universidade Federal do Rio Grande do Sul & RS & Brasil \\
\hline Melissa de Mattos Pimenta & Universidade Federal do Rio Grande do Sul & RS & Brasil \\
\hline Monika Weronika Dowbor & Universidade do Vale do Rio dos Sinos & RS & Brasil \\
\hline Myanna Lahsen & Instituto Nacional de Pesquisas Espaciais & SP & Brasil \\
\hline Oswaldo Martins E. do Amaral & Universidade Estadual de Campinas & SP & Brasil \\
\hline Pablo Quintero & Universidade Federal do Rio Grande do Sul & RS & Brasil \\
\hline Patrícia Mattos & Universidade Federal de São João Del-Rei & MG & Brasil \\
\hline Patricia Bandeira de Melo & Fundação Joaquim Nabuco & PE & Brasil \\
\hline Paula da Luz Galrão & Universidade Federal do Vale do São Francisco & PE & Brasil \\
\hline Paula Marcelino & Universidade de São Paulo & SP & Brasil \\
\hline Paulo Fonseca & Universidade Federal de Santa Catarina & SC & Brasil \\
\hline Paulo Peres & Universidade Federal do Rio Grande do Sul & RS & Brasil \\
\hline Pedro Feliú Ribeiro & Universidade de São Paulo & SP & Brasil \\
\hline Pedro Robertt & Universidade Federal de Pelotas & RS & Brasil \\
\hline Rafael Bennertz & Instituto Federal de Educação, Ciência e Tecnologia do RS & RS & Brasil \\
\hline Rafael de Almeida Evangelista & Universidade Estadual de Campinas & SP & Brasil \\
\hline Raquel Velho & Rensselaer Polytechnic Institute & & EUA \\
\hline Raquel Weiss & Universidade Federal do Rio Grande do Sul & RS & Brasil \\
\hline Rebecca Abers & Universidade de Brasília & DF & Brasil \\
\hline Riccardo Cappi & Universidade Estadual de Feira de Santana & BA & Brasil \\
\hline
\end{tabular}




\begin{tabular}{|c|c|c|c|}
\hline Richard Miskolci & Universidade Federal de São Paulo & SP & Brasil \\
\hline Roberta de Sousa Melo & Universidade Federal do Vale do São Francisco & PE & Brasil \\
\hline Roberto Dutra & Universidade Estadual do Norte Fluminense Darcy Ribeiro & RJ & Brasil \\
\hline Rodolfo Scachetti & Universidade Federal de São Paulo & SP & Brasil \\
\hline Rodrigo da Rosa Bordignon & Universidade Federal de Santa Catarina & SC & Brasil \\
\hline Rodrigo Ghiringhelli de Azevedo & Pontifícia Universidade Católica do Rio Grande do Sul & RS & Brasil \\
\hline Sadi Dal Rosso & Universidade de Brasília & DF & Brasil \\
\hline Salvador Schavelzon & Universidade Federal de São Paulo & SP & Brasil \\
\hline Sílvio Cesar Camargo & Universidade Estadual de Campinas & $\mathrm{SP}$ & Brasil \\
\hline Simone Magalhães Brito & Universidade Federal da Paraíba & PB & Brasil \\
\hline Soraya Vargas Cortês & Universidade Federal do Rio Grande do Sul & RS & Brasil \\
\hline Stelio Marras & Universidade de São Paulo & SP & Brasil \\
\hline Stephen Hilgartner & Cornell University & & EUA \\
\hline Susanne Lettow & Freie Universität Berlin & & Alemanha \\
\hline Tania Maria Cezar Hoff & Escola Superior de Propaganda e Marketing & SP & Brasil \\
\hline Tatiana Landini & Universidade Federal de São Paulo & SP & Brasil \\
\hline Telma Menicucci & Universidade Federal de Minas Gerais & MG & Brasil \\
\hline Thales Speroni Pereira da Cruz & Universidade Federal do Rio Grande do Sul & RS & Brasil \\
\hline Thays Mossi & Universidade Federal do Rio Grande do Sul & RS & Brasil \\
\hline Thiago Aparecido Trindade & Universidade de Brasília & DF & Brasil \\
\hline Tiago Duarte & Universidade de Brasília & DF & Brasil \\
\hline Tiago Jacaúna & Universidade Federal do Amazonas & $\mathrm{AM}$ & Brasil \\
\hline Tiago Santos Pereira & Universidade de Coimbra & & Portugal \\
\hline Vanessa Marx & Universidade Federal do Rio Grande do Sul & RS & Brasil \\
\hline Vanessa Rampton & McGill University & & Canadá \\
\hline Wilson Fusco & Fundação Joaquim Nabuco & $\mathrm{PE}$ & Brasil \\
\hline
\end{tabular}




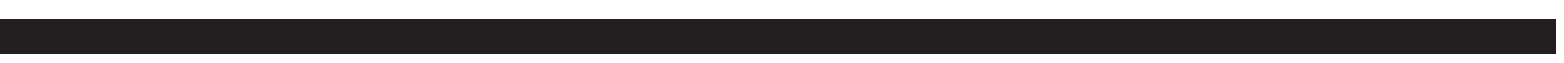

